\title{
Metal-organic Frameworks as a Robust Biocatalysis Platform for Enzymatic Production of Biodiesel
}

\author{
Xinlong Liu ${ }^{1}$, Peng $\operatorname{Liu}^{1} *, \mathrm{Na} \mathrm{An}^{1}$, Chen Liu ${ }^{1}$ \\ ${ }^{1}$ School of Biological Engineering, Tianjin Modern Vocational Technology College, Tianjin, 300350, China
}

\begin{abstract}
Metal-organic frameworks (MOFs) are an attractive class of hybrid materials with metal clusters and organic linkers. The unusual properties of MOFs, such as permanent nanoscale porosity, high surface area, uniformly structured cavities and the availability of in-pore functionality and outer-surface modification, are advantageous using as lipase immobilization platform. Herein, we covalent immobilized CALB onto MOFs and then evaluated the biocatalyst performance in the esterification of oleic acid with methanol for biodiesel production. Experimental data about the methanolysis process was evaluated by response surface methodology. The highest yield of $98.9 \pm 0.4 \%$ was obtained under the optimized conditions: methanol/oil ratio of 3.65:1, a reaction temperature of $46.3{ }^{\circ} \mathrm{C}$, a CALB@MOF loading of $117.77 \mathrm{mg}$ and a reaction time of $11.55 \mathrm{~h}$, which was closed to the predicted value (100.00\%). Verification experiment confirmed the validity of the predicted model.
\end{abstract}

\section{Introduction}

Biodiesel, series of fatty acid alkyl esters derived from vegetable oils and animal fats, can be used as a good alternative fuel for diesel engines owing to its environmental advantages and reproducibility ${ }^{[1]}$. The method of producing biodiesel mainly includes chemical catalysis and enzyme catalysis of esterification and esterification between oil and short chain alcohol. Compared with other methods, enzymatic biodiesel synthesis has many advantages, such as economy and environmental protection, which has attracted widespread attention. However, the high cost, poor reusability, and low stability of enzymes (lipase) hinder their wide application in industrial production process. Immobilization of enzyme is an efficient way to solve above problems, which requires an excellent material as a carrier.

Metal organic framework (MOFs) is a kind of porous crystal materials forming by the coordination combination of metal node and organic ligand ${ }^{[2]}$. Due to the diversity of metal types, organic ligand types and binding methods contained in MOFs, MOFs can have special functions to meet the application in different fields, such as catalysis, sensing, material storage and so on. One of the hot spots is co-assembling biocatalyst into nano-micro structure of MOFs to enhance the stability of biocatalyst, effectively improve the efficiency of chemical synthesis and the selectivity of enzyme catalysis.

In this work, we develop a synthetic route to immobilize Candida antarctica lipase B (CALB) into the bio-based MOF with adenine as the organic ligand based on the concept of biomimetic assembly. Then the resulted biocatalyst was tested for catalyse biodiesel production to explore their applicability. The optimum synthesis conditions were studied through response surface methodology (RSM).

\section{Materials and Methods}

\subsection{Material}

Candida antarctica lipase B was purchased from Beijing Cliscent Technology Co., Ltd. (Beijing, China). Zinc acetate, adenine, oleic acid and methanol were obtained from Shanghai Aladdin Bio-Chem Technology Co., LTD (Shanghai, China). Other chemicals of analytical grade were all purchased from Damao Chemical Reagent Factory (Tianjin, China).

\subsection{Immobilization of CALB}

The synthesis of MOF and immobilization of CALB were carried out simultaneously, and the detailed steps were as follows: $50 \mathrm{mg}$ of CALB and $200 \mathrm{mg}$ of Zinc acetate were added to $20 \mathrm{~mL}$ of deionized water and then mixed with 5 $\mathrm{mL}$ of boiling water containing $500 \mathrm{mg}$ of adenine. The system was incubated at room temperature for $48 \mathrm{~h}$. After that, the precipitate was collected by centrifugation (10000 r/min) and washed three times with deionized water. Finally, the obtain solid (named CALB@MOF) was freeze-dried and stored at $4 \circ \mathrm{C}$ until it was used.

The CALB loading in CALB@MOF was calculated using the following equation:

$$
C A L B \text { Loading }=\left(A-\frac{A_{1}}{B}\right) \times 100 \%
$$


Where $\mathrm{A}=$ total mass of $\mathrm{CALB}, \mathrm{A} 1=$ the total mass of the CALB in the supernatant and the detergent ${ }^{[3]}, B=$ the mass of the obtained CALB@MOF.

\subsection{Synthesis of Biodiesel}

Biodiesel was synthesized by the esterification of oleic acid and methanol catalysed by CALB@MOF. The reaction was performed in $15-\mathrm{mL}$ pressure flasks, containing $20 \mathrm{~mL}$ of oleic acid and $100 \mathrm{mg}$ of CALB@MOF. Then the mixture was homogenized with an ultrasonic cell disruptor (BILON-250Y) for $1 \mathrm{~min}$, operating at $12 \mathrm{~W}$ with 1 -s intervals every $3 \mathrm{~s}$. After adding methanol to the mixture, the flasks were incubated at certain temperatures and shaken in a thermostatic shaking water bath $(250 \mathrm{r} / \mathrm{min})$ for a certain time. The sample was prepared composed of the reaction mixture (part of the supernatant after centrifugation), n-hexane and salicylate acid methyl ester, mixed at a volume ratio of $1: 1: 2$, and each sample was tested three times to obtain accurate results.

The biodiesel yield was quantitatively analysed by gas chromatography (Beifen-Ruili Analytical Instrument Co., Beijing, China) equipped with an FID and SE-30 capillary column $^{[4]}$. Nitrogen gas was used as the carrier, and the flowrate was $20 \mathrm{~mL} / \mathrm{min}$. The initial GC column temperature was set at $160 \circ \mathrm{C}$ and heated to $220{ }^{\circ} \mathrm{C}$ at 15 ${ }^{\circ} \mathrm{C} \min ^{-1}$ after $2 \mathrm{~min}$. Then, the temperature rose to $260{ }^{\circ} \mathrm{C}$ within $5 \mathrm{~min}$ and was maintained for $10 \mathrm{~min}$. The temperature of the sampler and the detector temperature were set to $230{ }^{\circ} \mathrm{C}$ and $280^{\circ} \mathrm{C}$, respectively.

The biodiesel production process was analysed using CCD-RSM providing by Design Expert 8.0.6. A fourfactor-five-level with 30 experiments were designed in this study. The center points repeating 6 times were to measure the experimental pure error and enhanced the accuracy of data. The four factors their level values based on our previous research were A: molar ratio of methanol to oil $(2: 1,3: 1,4: 1,5: 1,6: 1)$, B:temperature $\left(40^{\circ} \mathrm{C}, 45^{\circ} \mathrm{C}\right.$, $\left.50{ }^{\circ} \mathrm{C}, 55^{\circ} \mathrm{C}, 60{ }^{\circ} \mathrm{C}\right), \mathrm{C}$ : CALB@MOF dosage $(80 \mathrm{mg}$, $100 \mathrm{mg}, 120 \mathrm{mg}, 140 \mathrm{mg}, 160 \mathrm{mg})$ and D: time (6 h, $8 \mathrm{~h}$, $10 \mathrm{~h}, 12 \mathrm{~h}, 14 \mathrm{~h}$ ) (Table 1). The 30 experiments were randomly conducted with the intention to minimize errors from the systematic trends.

Table 1 Factors and levels of central complex method

\begin{tabular}{ccccccc}
\hline Code & Variables & -2 & -1 & 0 & +1 & +2 \\
\hline A & $\begin{array}{c}\text { Molar ratio of } \\
\text { ethanol to oil }\end{array}$ & 2 & 3 & 4 & 5 & 6 \\
B & $\begin{array}{c}\text { Temperature }\left({ }^{\circ} \mathrm{C}\right) \\
\text { CALB@MOF }\end{array}$ & 40 & 45 & 50 & 55 & 60 \\
$\mathrm{C}$ & 80 & 100 & 120 & 140 & 160 \\
$\mathrm{D}$ & $\begin{array}{c}\text { dosage }(\mathrm{mg}) \\
\text { Time }(\mathrm{h})\end{array}$ & 6 & 8 & 10 & 12 & 14 \\
\hline
\end{tabular}

\section{Results}

\subsection{Development of regression model}

The relationship between biodiesel yield and four process parameters, i.e., molar ratio of methanol to oil, temperature, CALB@MOF dosage and time were optimized by using central composite design (CCD) of response surface methodology. Experiments containing 6 same center points, 8 axial points, and 16 factorial points were designed and the results were shown in Table 2 . As can be seen, the biodiesel yield varied from $40.70 \%$ to $98.00 \%$ and was strongly affected by the variation of the four conditions. Through the Analysis of Variance (ANOVA) of the experimental results, a second order polynomial regression equation (Equation 1) was established to describe the yield of biodiesel (Y) as a function of the molar ratio of methanol to oil (A), temperature (B), CALB@MOF dosage (C) and time (D). The positive coefficient of the item had a positive effect on yield increasing, while negative sign accounted for antagonistic effect.

Table 3 showed the results of ANOVA, which could be employed to evaluate the significance of the model and each item as well as their interaction on biodiesel yield. All the items except AD had a p-value less than 0.05 suggesting they were significant for the biodiesel production. Insignificant "lack of fit" $(p=0.1602)$ indicated that the fitting between model and experimental data was satisfactory. The value of determination coefficient $\left(\mathrm{R}^{2}\right)$ and adjusted determination coefficient $\left(\right.$ Adj- $\left.\mathrm{R}^{2}\right)$ approaching 1 both showed the better aptness of the model. Meanwhile, the "Pred- $\mathrm{R}^{2}$ " of 0.9901 was in

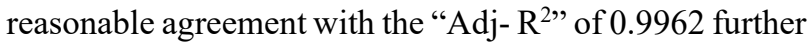
suggested that the equation had better regression and fitting degree. Therefore, the quadratic equation was suitable to analyse the experimental data and could be used to make predictions about the response for given levels of each factor.

$$
\begin{aligned}
& \text { Yield }(\%)=-1206.42167+103.775 A+ \\
& 32.83258 B+2.19448 C+30.40958 D-1.01125 A B+ \\
& 0.15031 A C-0.13 A D-0.00663 B C-0.33838 B D- \\
& 0.038156 C D-9.77792 A^{2}-0.25462 B^{2}- \\
& 0.0074 C^{2}-0.28855 D^{2}
\end{aligned}
$$

\subsection{Response surface plots for biodiesel production}

The 3D surface curves of the response (biodiesel yield) against any two independent variables were plotted to measure the interaction between two variables and determine the optimum conditions for maximum yield. Figure 1a illustrated the cumulative effect of the molar ratio of methanol to oil and temperature meanwhile keeping other factors at the " 0 " level. It showed that the biodiesel yield reduced when the molar ratio of methanol to oil was increased or decreased beyond the central level. Low level of molar ratio limited biodiesel yield due to insufficient substrates. While excessive methanol at a high level of molar ratio would break the molecular structure of the CALB, resulting in its loss of catalytic capacity. The increment of temperature initially led to a slight increase of biodiesel yield and then rapidly decline at the higher level of temperature. Figure $1 \mathrm{~b}$ showed the interaction effect between molar ratio of methanol to oil and CALB@MOF dosage toward biodiesel yield. It was 
believed that the yield of biodiesel decreased with the increase of the molar ratio of methanol to oil. On the contrary, the yield was increased with the increase of the CALB@MOF dosage, but the trend of growth was decreasing. The more catalysts, the faster the reaction. Figure 1c reveals the variation of yield with temperature and CALB@MOF dosage. The maximum yield should occur near the central level of the temperature. The

Table 2 Experimental design and results of central complex method

\begin{tabular}{cccccc}
\hline NO. & A & B & C & D & Yield \% \\
\hline 1 & 3 & 55 & 140 & 8 & 88.38 \\
2 & 6 & 50 & 120 & 10 & 40.70 \\
3 & 5 & 45 & 140 & 8 & 80.66 \\
4 & 3 & 45 & 100 & 12 & 88.63 \\
5 & 4 & 50 & 160 & 10 & 94.90 \\
6 & 5 & 45 & 100 & 8 & 57.62 \\
7 & 5 & 55 & 140 & 8 & 67.93 \\
8 & 4 & 40 & 120 & 10 & 79.66 \\
9 & 4 & 50 & 120 & 10 & 96.80 \\
10 & 4 & 50 & 80 & 10 & 73.59 \\
11 & 4 & 50 & 120 & 10 & 94.97 \\
12 & 5 & 55 & 100 & 8 & 47.59 \\
13 & 4 & 50 & 120 & 14 & 98.00 \\
14 & 3 & 45 & 140 & 8 & 81.33 \\
15 & 4 & 50 & 120 & 10 & 96.70 \\
16 & 3 & 55 & 140 & 12 & 87.50 \\
17 & 5 & 55 & 140 & 12 & 66.09 \\
18 & 4 & 50 & 120 & 10 & 96.10 \\
19 & 3 & 55 & 100 & 12 & 85.00 \\
20 & 3 & 45 & 140 & 12 & 93.25 \\
21 & 4 & 60 & 120 & 10 & 61.60 \\
22 & 5 & 45 & 140 & 12 & 93.09 \\
23 & 5 & 45 & 100 & 12 & 75.10 \\
24 & 3 & 45 & 100 & 8 & 69.55 \\
25 & 4 & 50 & 120 & 10 & 96.50 \\
26 & 4 & 50 & 120 & 6 & 83.95 \\
27 & 3 & 55 & 100 & 8 & 79.20 \\
28 & 5 & 55 & 100 & 12 & 51.30 \\
29 & 4 & 50 & 120 & 10 & 95.70 \\
30 & 2 & 50 & 120 & 10 & 73.26 \\
\hline & & & & &
\end{tabular}

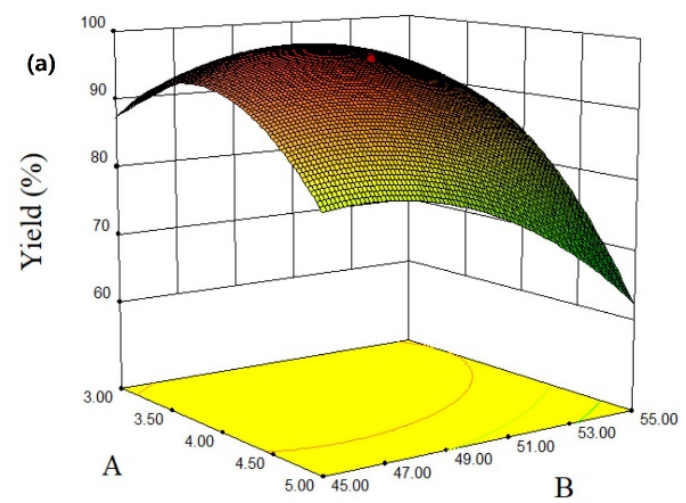

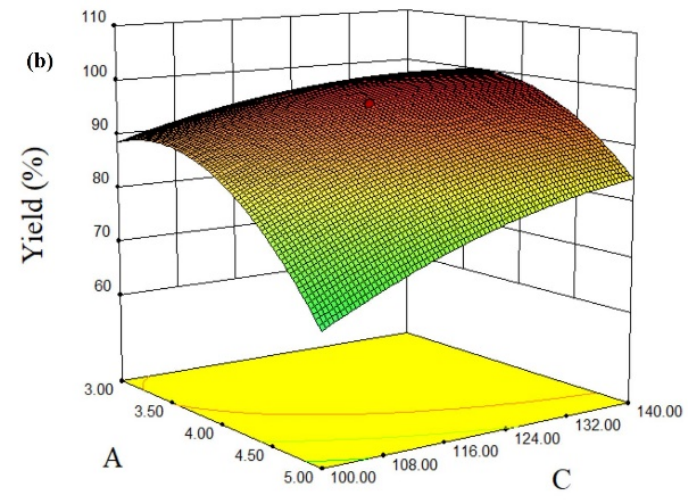
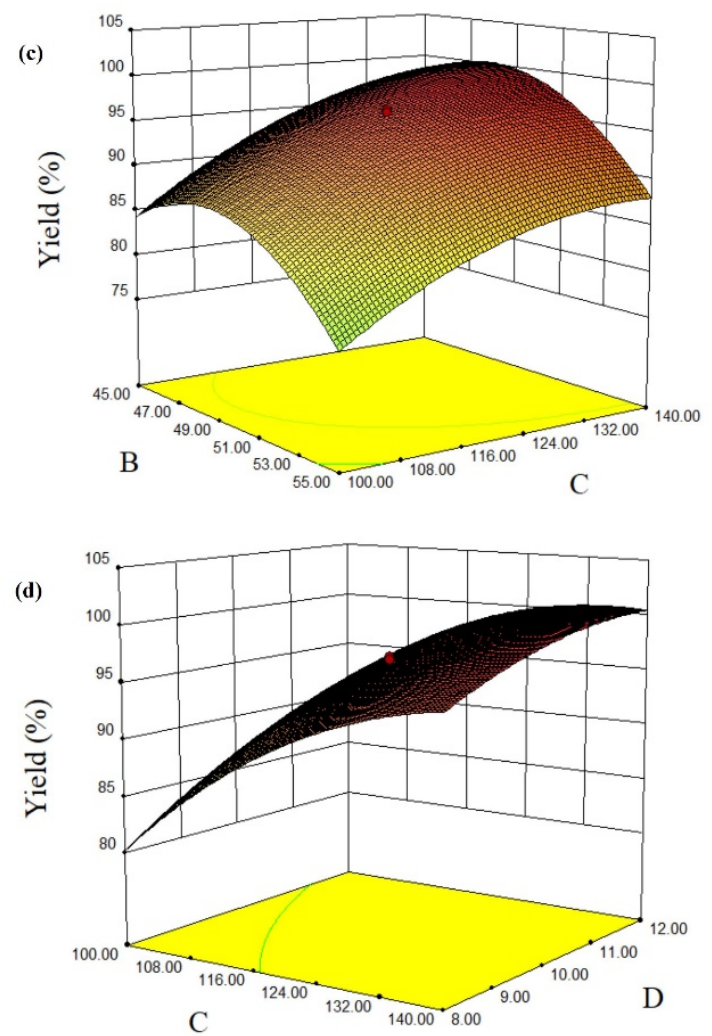

Fig. 13 D surfaces of the combined effects of two variables on the yield of biodiesel (a) interaction between molar ratio of methanol to oil and CALB@MOF dosage; (b) interaction between molar ratio of methanol to oil and Time; (c) interaction between Temperature and Time; (d) interaction between CALB $a$ MOF dosage and Time.

Table 3 ANOVA for the yield of biodiesel

\begin{tabular}{ccccc}
\hline Terms & Sum of squares & F-value & P-value (Prob $>$ F) & \\
\hline Model & 7501.62 & 546.44 & $<0.0001$ & Significant \\
A : Molar ratio of methanol to & 1642.88 & 1675.41 & $<0.0001$ &
\end{tabular}




\begin{tabular}{|c|c|c|c|c|}
\hline B : Temperature & 436.35 & 444.99 & $<0.0001$ & \\
\hline C : CALB@MOF dosage & 898.28 & 916.06 & $<0.0001$ & \\
\hline D : Time & 382.53 & 390.10 & $<0.0001$ & \\
\hline $\mathrm{AB}$ & 409.04 & 417.14 & $<0.0001$ & \\
\hline $\mathrm{AC}$ & 144.52 & 147.38 & $<0.0001$ & \\
\hline $\mathrm{AD}$ & 1.08 & 1.10 & 0.3105 & \\
\hline $\mathrm{BC}$ & 7.04 & 7.18 & 0.0173 & \\
\hline $\mathrm{BD}$ & 183.23 & 186.86 & $<0.0001$ & \\
\hline $\mathrm{CD}$ & 37.26 & 37.99 & $<0.0001$ & \\
\hline $\mathrm{A}^{2}$ & 2622.39 & 2674.31 & $<0.0001$ & \\
\hline $\mathrm{B}^{2}$ & 1111.33 & 1133.33 & $<0.0001$ & \\
\hline $\mathrm{C}^{2}$ & 240.59 & 245.85 & $<0.0001$ & \\
\hline $\mathrm{D}^{2}$ & 44.88 & 45.77 & $<0.0001$ & \\
\hline Residual & 14.73 & & & \\
\hline Lack of Fit & 12.29 & 2.52 & 0.1602 & Insignificant \\
\hline Pure error & 2.44 & & & \\
\hline Std. Dev. & 0.99 & \multicolumn{2}{|c|}{$\mathrm{R}^{2}$} & 0.9980 \\
\hline Mean & 79.82 & \multicolumn{2}{|c|}{ Adj- $R^{2}$} & 0.9962 \\
\hline C.V.\% & 1.24 & \multicolumn{2}{|c|}{ Pre- $R^{2}$} & 0.9901 \\
\hline PRESS & 74.28 & \multicolumn{2}{|c|}{ Adeq Precision } & 83.532 \\
\hline
\end{tabular}

CALB $@$ MOF dosage could promote the increase of biodiesel yield. The influence of CALB@MOF dosage and time on biodiesel yield was shown in Figure 1d. Increasing the reaction time affected the yield a little, which might be due to the reaction being near equilibrium and the substrate being exhausted.

\subsection{Validation of the model}

Based on the CCD and ANOVE results, equation (1) was employed to determine the optimum conditions composition of biodiesel yield, which was $100 \%$ at a molar ratio of methanol to oil of $3.65: 1$, a reaction temperature of $46.3^{\circ} \mathrm{C}$, a CALB@MOF loading of 117.77 $\mathrm{mg}$ and a reaction time of $11.55 \mathrm{~h}$. Under the optimal conditions, a validated experiment repeated three times was conducted to verify the predictions. The actual yield was $98.9 \pm 0.4 \%$ showing an agreement with the regress model prediction. Thus, the almost identical results could confirm that the CCD model was accurate and reliable for predicting the biodiesel yield within the range of parameters selected by the experimental design.

\section{Conclusion}

In the current research, CALB was successfully immobilized into a biology-based MOF system with adenine as an organic ligand by bionic assembly. Compared with traditional immobilized enzyme, the synthesis process of CALB@MOF was simple, green and pollution-free.CALB@MOF had demonstrated excellent performance in catalytic synthesis of biodiesel from oleic acid and methanol. A central composite design of response surface methodology were used to study the effects of four factors, molar ratio of methanol/oil, temperature, CALB@MOF dosage and time on the biodiesel yield. The maximum yield of biodiesel reached $98.9 \pm 0.4 \%$ under optimal reaction conditions (molar ratio of methanol to oil of $3.65: 1$, temperature of $46.3{ }^{\circ} \mathrm{C}$, CALB@MOF loading of $117.77 \mathrm{mg}$ and reaction time of
$11.55 \mathrm{~h}$ ), which was in agreement with the predicted value by regress model.

\section{References}

1. B.L. Salvi and N.L. Panwar. Renew. Sust. Energ. Rev. 16, 3680-3689 (2012)

2. J. Huo, J. Aguilera-Sigalat, S. El-Hankari and D. Bradshaw. Chem. Sci. 6, 1938-1943 (2015)

3. J. Gao, W. Kong, L. Zhou, Y. He, L. Ma, Y. Wang, L. Yin and Y. Jiang. Chemical Engineering Journal 309, 70-79 (2017)

4. H. Gu, Y. Jiang, L. Zhou and J. Gao. Energy Environ. Sci. 4, 1337-1344 (2011) 